\title{
Mucoadhesive microspheres of chitosan and polyvinyl alcohol as a carrier for intranasal delivery of insulin: in vitro and in vivo studies
}

\begin{abstract}
The aim of this study was to investigate the capabilities of chitosan microspheres as drug carrier system in co-formulation with polyvinyl alcohol (PVA), to improve the systemic absorption of intranasal insulin delivery.

Insulin loaded microspheres was developed from varying ratio of chitosan solutions along with polyvinyl alcohol (PVA) as additive polymer using spray drying method. Different formulations were developed and morphological studies of the optimized formulas showed that the size range of spherical shaped particulate matters existed from $200 \mathrm{~nm}$ to $2 \mu \mathrm{m}$. It was clearly observed that physicochemical properties of the microspheres were extensively affected by changing the concentration ratio of the two polymeric materials. In vitro studies of insulin release pattern was performed in various time intervals up to $24 \mathrm{~h}$. It was evident that microspheres made up of chitosan showed initial burst release but slower release as the experiment continued. Microspheres made up of combination of the aforementioned two polymers had instant, sharp and burst drug release. Surprisingly there was no absorption after intranasal delivery of chitosan-PVA microspheres in groups of rats comparing to formulated chitosan microspheres having profound absorption due to their smaller particle size, slower drug release rate and better mucoadhesive properties. Therefore, significant reduction in the plasma blood glucose level for chitosan based optimized formulation was seen right after 4.5 hours compared with control group. The aim of the present study was to fabricate an appropriate application of polymeric microspheres for intranasal delivery of insulin using a novel optimized formulation based on industrial level spray drying technique and to realize the possible barriers in scale up process of its large scale production, considering the effectiveness of polyvinyl alcohol (PVA) and chitosan to increase mucoadhesivness, gelling ability and ultimately effective release behavior pattern of insulin. According to this study, the combination of the polymers used and the mean particle size of formulated microspheres were found to be key factors in insulin drug release resulting for further enhancement of insulin absorption via intranasal route of delivery.
\end{abstract}

Keywords: intranasal delivery, microspheres, blood glucose level, absorption enhancement, mucoadhesive polymers, chitosan, polyvinyl alcohol
Volume 3 Issue 2 - 2017

\author{
Samira Nasiri Zadeh,' Saeid Rajabnezhad,' \\ Majid Zandkarimi,' Somayeh Dahmardeh,' \\ Leyla Mir,' Mohammad Ali Darbandi,' \\ Mohammad Reza Rajabnejad ${ }^{3}$ \\ 'Zabol University of Medical Sciences, Iran \\ ${ }^{2}$ Department of Biochemistry, Sussex University, UK \\ ${ }^{3}$ Department of History of Medical Sciences, Iran University of \\ Medical Sciences, Iran
}

Correspondence: Mohammad Ali Darbandi, Analytica Research Laboratory, School of pharmacy, Zabol University of Medical Sciences, Zabol, Iran, Tel +98 542 22535278, Fax +98 5422253528,Email alidarbani@yahoo.com

Received: September 04, 2016 | Published: March 23, 2017
Abbreviations: PVA, polyvinyl alcohol; IV, intravenous; IM, intramuscular; GI, gastrointestinal; MW, molecular weight; SEM, scanning electron microscopy; AUC, area under curve; MDT, measurement of mean dissolution time; SD, standard deviation.

\section{Introduction}

Diabetic mellitus is a chronic disease that appears as result of absolute or partial deprivation of insulin. It constitutes a group of metabolic disorders which is common in hyperglycaemic patients. It would be regarded as an imminent cause of disease attributed to more than $25 \%$ of the blindness compared to non-diabetic patients. Insulin is a peptide hormone which regulates the metabolism of carbohydrates and fats by promoting the absorption rate of glucose level within the cells. It has been shown that absorption of peptides and proteins by the oral route is quite low or even negligible based owing to their molecular weight, polarity and lipophilicity of the drugs, enzymatic degradation and instability etc. Hence administration of insulin like macromolecules are generally carried out by parenteral therapy i.e., subcutaneous or intramuscular routes of delivery rather than oral route. ${ }^{1,2}$
Invasiveness of the Intravenous (IV) or intramuscular (IM) injections as well as the least patient compliance compelled scientists either to modify the previously existed dosage forms which are already in the market to prevent them from gastrointestinal (GI) degradations or to explore other possible route for drug delivery. Apart from oral or injection therapies, various other means for insulin delivery has been already investigated including, transdermal, buccal, nasal, pulmonary, ocular and rectal delivery. ${ }^{3}$ Intranasal route has traditionally been practiced for centuries in the treatment of various local diseases such as nasal congestion, sinusitis, allergic, rhinitis and infectious illnesses. In the last few decades, treatment of disease once a rapid onset of action is desired have been more emphasized such as migraine, severe pains, menopausal symptoms and asthma like attacks.

Intranasal delivery is a safe route of drug administration providing vast surface area for drug to be absorbed with higher degree of patient compliance compared to parenteral routes. Large surface area and presence of wide mucociliary network like structures are key parameters for intranasal drug delivery. They directly influence the entrance of drug molecules into the systemic circulation after being absorbed depending on lipophicity of drug molecular weight $(\mathrm{Mw})$. 
Furthermore, avoidance of hepatic first pass metabolism makes it a preferred route for the delivery of small drug molecules but not basically the macromolecules as the permeability and degradability are rate-limiting steps in their systemic absorptions.

Feasibility of intranasal route for delivery of peptides and proteins has already been evaluated as mentioned before, due to the perceived less demanding barriers to peptides and proteins transport across the nasal epithelium as compared to the oral mucosa and nasal cavity comprising lower enzymatic content, higher leakiness of the membrane and high vascularity and no hepatic first pass metabolism But still there are few nasal peptide based products which have appeared on the market e.g. calcitonin (Miacalcin ${ }^{\circledR}$, Fortical ${ }^{\circledR}$ ), desmopressin $\left(\right.$ Desmospray $\left.^{\circledR}\right)$, nafarelin $\left(\right.$ Synarel $\left.^{\circledR}\right)$ buserelin $\left(\right.$ Suprecur $\left.^{\circledR}\right)$ and oxytocin $\left(\right.$ Syntocinon $\left.^{\circledR}\right), 4,5$

These products do not contain absorption enhancers and hence would show low bioavailability but are effective enough due to the low plasma levels needed to obtain a therapeutic effect.

As indicated above, one of the demerits of intranasal administration of peptides and proteins is their low bioavailability. ${ }^{6}$ This can be attributed to their intrinsic chemical structure as well as their physicochemical properties such as hydrophilicity, high $\mathrm{Mw}$ and intrinsic instability in the presence of enzymes. Owing to their physicochemical properties, peptides and proteins are expected to pass the nasal membrane via the paracellular pathways more specifically via the tight junctions. However, the diameter of the tight junctions in the nasal cavity are ranged from $3.9-8.4 \AA^{7}$ and hence the transport through these junctions would be limited or considered to be negligible for larger drug molecules, such as insulin with a structural diameter of $26.8 \AA$ in the monomeric form. ${ }^{8}$ It has already been shown by Costantino et al. ${ }^{9}$ that permeation enhancers were able to open the tight junctions in the nasal mucosa to about 10-15 times higher, approaching a maximal diameter in the order of $15 \mathrm{~nm} .{ }^{9}$ The application of absorption enhancers were often studied in mucoadhesive like formulations in the form of liquids, gelling systems, nanoparticles and microspheres by few authors to better approach the transport of peptides and proteins across the nasal mucosa. ${ }^{10-16}$ Of special interest has been the use of chitosan as an absorption enhancer and a bioadhesive materials acting by opening the tight junctions of nasal mucosa to overcome the high degree effect of the mucociliary clearance by increasing the residence time of the formulation in the nasal cavity. ${ }^{17}$

Chitosan, a natural linear polycationic, biocompatible, biodegradable and mucoadhesive polymer derives from alkaline deacetylation of chitin of several Mw $(10 \mathrm{kDa}-2000 \mathrm{kDa})$ with varying degrees of deacetylation (40-98\%). The inherent characteristics of the chitosan determines physicochemical properties of the polymer which are key for its effectiveness as an absorption enhancer as well as being a mucoadhesive material. Chitosan is positively charged in acidic $\mathrm{pH}$, where it forms a water soluble salt. The positive charges interact with the sialic acid in the mucus and makes it vulnerable to disrupt the tight junctions by translocation of the membrane and cytocylic proteins into the cells cytosol, effectively increasing the systemic absorption of proteins and peptides. ${ }^{18}$ Its apparent $\mathrm{pKa}$ is dependent on the degree of deacetylation, but generally is in the order of $6.3 .{ }^{17}$ Application of chitosan microspheres for nasal delivery for drug molecules such as morphine, ${ }^{19}$ zolmitriptan, ${ }^{20}$ verapamil, ${ }^{21}$ metoclopramide, ${ }^{22}$ insulin, ${ }^{23-25}$ goserelin ${ }^{26}$ and human growth hormone ${ }^{13}$ using different production methods i.e., spray drying ${ }^{20}$ and emulsification techniques ${ }^{24}$ have already been studied. They showed that chitosan in solution enables the transport of even macro drug molecules across the nasal membrane. Moreover, application of chitosan in microspheric form enhances transfusion of the macromolecules. On the other hand, PVA, a hydrophilic biocompatible polymeric excipient which is being widely used in biomedical and pharmaceutical applications. The polymer has unique gelling characteristics, which in turn makes it responsible for its adhesiveness and film-formation properties. PVA is used in the present study to investigate the effect of polymeric blend along with chitosan to explore the possibility for the improvement of mucoadhesive and drug release properties of insulin from the system either of which to be used alone or in combination with each other in appropriate ratios to understand if they could synergic mucoadhesiveness and drug release patterns. When PVA comes into contact with the physiological fluid, it increases the viscosity and forms thin hydrogel film like structure on nasal surface.

Mucoadhesive polyvinyl alcohol hydrogels produced by freezing/ thawing processes: applications in the development of wound healing systems. But the rate, degree and extent of absorption from PVA formed hydrogels needed to be studied more in detail which will be discussed in this paper further. It has been reported that such chitosan/ PVA blend, forms in situ gel once it comes in contact with nasal muciliary membrane and minimizes the nasal mucociliary clearance of the formulation. The blend has also been used by Minoura et al. (1998) \& Koyanoa et al. (2000) to study the surface properties and their relationship with the cell attachment or growth behaviour. Kim et al. (2002) has studied electrical charge sensitivity, Wang et al. (2004) for $\mathrm{pH}$ sensitivity and by Tang et al. (2007) for rheological characterization of the blend. ${ }^{25}$

The key point which has to be clearly emphasized is that very rarely feasibility of large scale production of industrial batches of microspheric sprayed dried micromolecules including insulin have been studies. Therefore one of our concerns in this paper was the industrial scale up of the optimized formulation with spray drying technique more in detail. To understand the possible obstacles ahead of large scale production, the experiments have been performed in a small laboratory scales primarily before scaling it up

Therefore, the purpose of the present study was to design an fabricate an appropriate and suitable applicable polymeric microspheres for intranasal delivery of insulin using a novel optimized formulation based on industrial level spray drying technique and to realize the possible barriers in scale up process of its large scale production, considering the effectiveness of polyvinyl alcohol (PVA) and chitosan to increase mucoadhesivness, gelling ability and ultimately effective release behavior pattern of insulin. It is worthy to state that spray dried technique has been studied in accordance of being a well-defined means for industrialization. Moreover, it was estimated that Spray dried insulin particles would retain biological activity of slow in-vitro assay/ The effect of process variables on the degradation and physical properties of spray dried insulin intended for inhalation. ${ }^{26}$

The designed and produced microspheres were further characterized and intranasal absorption of insulin encapsulated in the polymeric matrix was evaluated in vitro and in vivo after intranasal administration.

\section{Materials and methods}

\section{Materials}

Dry powder recombinant human insulin, medium molecular weight chitosan (200-800 cP) and polyvinyl alcohol (PVA) 40kDa 
Mw were all obtained from Sigma-Aldrich (USA). Recombinant Regular Human Insulin 100 I.U was purchased from Exir (Iran), monobasic potassium phosphate, glacial acetic acid and acetonitrile were also purchased from Merck (Darmstadt, Germany). Rest of the chemicals which have been used in this study were of high analytical grade.

\section{Methods}

Preparation of standard solution: Concentrated solution of insulin was prepared by dissolving $30 \mathrm{mg}$ of insulin dry powder in $5 \mathrm{~mL} 0.1$ mole/ $\mathrm{L} \mathrm{HCl}$ acid, $25 \mathrm{~mL}$ of distilled water was added to the acidic solution and was mixed further in a mechanical stirrer. The final stock solution was diluted appropriately to prepare standard dilutions of different concentrations ranging from $0.5,0.25,0.125,0.0625,0.0312$ and $0.015 \mathrm{mg} / \mathrm{mL}$.

Preparation of the microspheres: Microspheres were prepared using spray drying technique for solutions containing insulin, chitosan and PVA at different ratios in $1 \%$ acetic acid as a solvent. The solutions were agitated for 10 minutes with a magnetic stirrer (Heidolph-D-91126, Germany) at speed of 1300rpm. Different composition of the prepared formulations is shown in Table 1. The concentration of insulin was kept constant by addition the fixed amount $(6 \mathrm{~mL})$ of Recombinant Human Insulin 100 IU solution in all the formulations i.e., each $1 \mathrm{IU}$ is equivalent to $0.0347 \mathrm{mg}$ of human insulin. Chitosan based microspheres were prepared by dissolving $100 \mathrm{mg}$ chitosan in $100 \mathrm{~mL}$ of $1 \% \mathrm{v} / \mathrm{v}$ acetic acid which were well stirred for $10 \mathrm{~min}$. Different concentrations of $0.5 \%$ and $0.25 \%(\mathrm{w} / \mathrm{v})$ were formed from the already prepared fresh solution by stirring at $1300 \mathrm{rpm}$. Fixed amount of Recombinant Human Insulin $(6 \mathrm{~mL})$ was added further drop wise using insulin injection syringe to the solutions while stirring on an agitator. PVA polymeric solutions were also prepared with the same method as described above. For this, $100 \mathrm{~mL}$ distilled water was heated up to about $70-75^{\circ} \mathrm{C}$ and the temperature was kept constant. 100 $\mathrm{mg}$ of PVA powder was added slowly to prevent the formation of agglomerates. It was then well stirred by the same speed to avoid the possible agglomeration of the polymer. Then solution was further stirred for another $10 \mathrm{~min}$ to obtain clear solution. Different compositions of the chitosan/PVA polymeric blend were prepared according to the ratios indicated in Table 2. The solutions were stirred at $1300 \mathrm{rpm}$ for 10 min and constant amount of Regular Human Recombinant Insulin 100 I.U. was added drop by drop using a syringe. The solutions were then dried using spray drying technique to obtained desired spherical microspheres of suitable particle size. For this, the spray dryer (Buchi 191 , Switzerland), was set to have the inlet temperature of $100^{\circ} \mathrm{C}$ and outlet temperature of $90^{\circ} \mathrm{C}$. Aspirator was $80 \%$ and pump setting was $8 \mathrm{~mL} / \mathrm{min}$. The obtained microspheres were collected and maintained in a desiccator for future analysis.

Table I Compositions of various formulations based on different concentrations of chitosan and polyvinyl alcohol

\begin{tabular}{lll}
\hline Group A, containing $100 \mathbf{~ m g}$ chitosan in $1 \%$ acetic acid (w/v\%) \\
\hline Formulation & $\begin{array}{l}\text { Chitosan } \\
\text { Amount }\end{array}$ & Composition (\%) \\
Al & $1000 \mathrm{mg}$ & $1 \%$ \\
A2 & $0.50 \%$ \\
A3 & $0.25 \%$
\end{tabular}

Table Continued..

Group A, containing 100 mg chitosan in $1 \%$ acetic acid (w/v\%)

\section{Group B, containing different ratios of chitosan: PVA}

$\begin{array}{lll}\text { Formulation } & \text { Chitosan: PVA } & \text { Amount of Chitosan: PVA (mg) } \\ \text { BI } & 50: 50: 00 & 500: 500 \\ \text { B2 } & 70: 30: 00 & 700: 300\end{array}$

Group C, containing only PVA (I\%)

Formulation PVAAmount

PVA Composition (w/v \% in Distilled water)

C

$1000 \mathrm{mg}$

$1 \%$

Table 2 Inter day variation of the method of analysis

\begin{tabular}{llllll}
\hline CV \% & Mean & \multicolumn{2}{l}{$\begin{array}{l}\text { Measured } \\
\text { concentration }(\mu \mathrm{g} / \mathrm{mL})\end{array}$} & $\begin{array}{l}\text { Real } \\
\text { concentration } \\
(\mu \mathrm{g} / \mathrm{mL})\end{array}$ \\
\hline & & Day3 & Day2 & Dayl & \\
0.78 & 0.508 & 0.509 & 0.503 & 0.512 & 0.5 \\
2.17 & 0.253 & 0.252 & 0.248 & 0.259 & 0.25 \\
2.43 & 0.123 & 0.127 & 0.123 & 0.121 & 0.125 \\
3.23 & 0.065 & 0.0677 & 0.064 & 0.064 & 0.0625 \\
& & & & &
\end{tabular}

\section{Characterization of microspheres}

\section{Measurement of encapsulation efficacy}

Encapsulation efficacy was determined by dispersing $10 \mathrm{mg}$ of each microspheres in $2.5 \mathrm{~mL}$ methanol: acetic acid (1:1) ratio. The obtained suspensions were kept on a rotary extractor overnight. The supernatant layer was taken out after $12 \mathrm{~h}$ without being centrifuged. It was further diluted with HPLC mobile phase composed of $45 \%$ acetonitrile and $55 \%$ buffer solution $\mathrm{pH} 7.5$ and was injected into the HPLC instrument for the analysis.

\section{Scanning electron microscopy}

Morphology of each sample was studied by Scanning Electron Microscopy (SEM) (Philips XL 30 scanning microscope, Philips, The Netherlands) at $25 \mathrm{KeV}$. The samples were placed onto an aluminum stub using double sided adhesive carbon disc (SCD005 Sputter coater, Bal-Tec, DE). The samples were sputter coated with gold $20 \mathrm{~mA}$ for 4 minute prior to analysis.

\section{HPLC chromatography}

Several methods such as capillary electrophoresis and gradient HPLC systems have been evaluated in order to overcome the need for gradient HPLC system for analysis of insulin. ${ }^{27,28}$ It is necessary to apply two or three pumps in gradient HPLC method. As stated before the mobile phase of the systems was composed of $45 \%$ acetonitrile and $55 \%$ buffer solution $\mathrm{pH}$ 7.5. The buffer solution was prepared fresh at the time of analysis by mixing $0.01 \mathrm{M}$ monobasic potassium phosphate and $\mathrm{KOH} 8 \mathrm{~N}$ solutions. The $\mathrm{KOH} 8 \mathrm{~N}$ solution was used for adjusting the buffer $\mathrm{pH}$ at 7.5. The analysis was carried out at a flow rate of $1 \mathrm{~mL} / \mathrm{min}$ with 1100 psi pressure. The instrument (HPLCCecil-CE 4104, England) was equipped to UV detector (Cecil-CE 
4300, England) and the measurement was carried out at $210 \mathrm{~nm}$ with ODS-3.5 $\mu \mathrm{m} 125 \times 4 \mathrm{~mm}$ column (Perfectsil Target, Germany). Phosphate buffer $\mathrm{pH} 7.5$ was produced by dissolving $6.8 \mathrm{~g}$ potassium dihydrogen phosphate in 1 liter of distilled water and the desired $\mathrm{pH}$ was adjusted to 7.5 using $8 \mathrm{~N}$ potassium hydroxide. The buffer solution was then filtered with syringe filter and was mixed with acetonitrile in ratio of 45:55. $4.5 \mathrm{mg}$ of standard insulin powder was dissolved in $9 \mathrm{~mL}$ of deionized distilled water to obtain the stock solution having concentration of $0.5 \mathrm{mg} / \mathrm{mL}$. Different concentrations of $0.25,0.125$ and $0.0625 \mathrm{mg} / \mathrm{mL}$ were further prepared from the stock solution. $50 \mu \mathrm{L}$ of each sample was then injected into the HPLC system using the aforementioned mobile phase at $210 \mathrm{~nm}$. The tests were repeated in triplicate.

\section{In vitro drug release pattern}

$125 \mathrm{~mL}$ of $0.2 \mathrm{M}$ potassium dihydrogen phosphate was mixed with $14 \mathrm{~mL}$ of $0.2 \mathrm{M}$ sodium hydroxide. The mixture was then volume up to $500 \mathrm{~mL}$ with distilled water to obtain buffer system solution having $\mathrm{pH} 6.5$ considered as a $\mathrm{pH}$ of the nasal mucosa. $30 \mathrm{mg}$ of each formulation was thoroughly weighted and were transferred to different labeled vials. $2 \mathrm{~mL}$ of the previously fresh prepared buffer solution was added to each vial. The vials were kept on a mechanical shaker and the sampling was performed at different time intervals of $0.16,0.33,0.5,0.66,0.83,1,2,3,4,5,12$ and $24 \mathrm{~h}$. Within each of the specific time periods, $0.5 \mathrm{~mL}$ of the solutions was taken out and immediately was replaced with the same fresh buffer system. Samples were then centrifuged at $10000 \mathrm{rpm}$ for $10 \mathrm{~min} .50 \mu \mathrm{L}$ of supernatant was injected into HPLC instrument equipped with UV visible detector having $125 \times 4 \mathrm{~mm}$ column in flow rate of $1 \mathrm{~mL} / \mathrm{min}$ and particles size of $3.5 \mu \mathrm{m}$. Composition of the mobile phase system was as described above and measurement was carried out at $210 \mathrm{~nm}$. Amount of insulin released was measured based on the obtained AUC of insulin chromatogram and the previously constructed calibration curve. The concentration and the amount of the drug released in various time intervals were calculated and ultimately cumulative percentage of drug release was plotted.

\section{Measurement of insulin release efficacy}

Trapezoidal method was used to calculate the release efficacy of insulin from each formulation. Release efficacy percentage was calculated using the following formula:

$$
\text { Release Efficacy percentage }=\frac{A U C}{A U C S} \times 100
$$

Where AUC is the area under curve of drug release chromatogram and AUCs is the area under curve of standard sample.

\section{Measurement of mean dissolution time (MDT)}

To measure mean dissolution time (MDT), trapezoidal method was used again by dividing the area under curve of the release diagram by final release percentage of each particular formulation.

\section{Animal studies}

24 female Wistar rats weighting from $200-250 \mathrm{~g}$ were obtained from the animal house of the faculty of pharmacy, Zabol University of Medical Science following the code of practice for the established university animal ethics. The Helsinki animal ethics law for evaluation of the absorption mechanism of insulin from the nasal mucosa was also considered.
All in vivo experiments were carried out according to the National Institute of Health guidelines for the care and use of laboratory animals and to the European Community Council Directive for the care and use of laboratory animals of 24 November 1986 (86/609/ EEC) and were approved by the local ethics committee.

The animals were kept in light and dark conditions for $24 \mathrm{~h}$, at mean temperature of $20-25^{\circ} \mathrm{C}$ with relative humidity of $45-65 \%$. They were further maintained fasted for 12 before initiation of the study having free access to water. The animals were classified into 4 different groups each containing 6 rats, anaesthetized in a saturated chloroform chamber $2 \mathrm{~h}$ before instillation of the formulations and were controlled every $1 \mathrm{~h}$ to make sure that the animals are still alive until the scheduled time is over. The control group (A), was composed of the animals that were administered $0.5 \mathrm{~mL}$ phosphate buffer $\mathrm{pH} 7.4$ via nasal route. Standard group (B), received fixed amount of insulin equivalent to $2 \mathrm{mg}$ of the Regular Human Insulin intranasally. $17 \mathrm{mg}$ of the two optimized formulations (A3 and B3) were suspended in $2 \mathrm{~mL}$ phosphate buffer $\mathrm{pH} 7.4$ and were then instilled using $2 \mathrm{~mL}$ syringe to the nasal cavities of the rats in groups $\mathrm{C}$ and $\mathrm{D}$ respectively. $0.5 \mathrm{~mL}$ of blood samples were taken out from the tail veins of the animals $2 \mathrm{~h}$ before initiation of the study and consequently at the time intervals of $0,1.5,3,4.5,6,7.5$ and $9 \mathrm{~h}$ after instillation of the optimized formulations. Blood samples were centrifuged at 10000rpm for 10 min to obtain clear and supernatant solution. The solutions were then filtered with $0.45 \mu \mathrm{m}$ syringe filter and the concentration of the glucose in the blood samples was measured using HPLC method after injection of appropriate dilutions i.e., $100 \mu 1$.

\section{Statistical analyses}

All the studies were performed in triplicate and data are presented as mean $\pm \mathrm{SD}$ (standard deviation). Statistical significance was analyzed using Student's t-test and one way ANOVA method. The $p$ value $<0.05$ was considered as significant.

\section{Results and discussion}

The loading percentage and release efficacy of insulin in three different groups of polymeric microspheres A, B and C has been shown in Table 3. Cumulative release profile of insulin from microspheres id different formulations was also depicted in Figure 1. Based on drug release profile and MDT data from Table 3, presence of PVA in the polymeric matrix of the microspheres increases the release rate of insulin from encapsulated polymeric matrix which is not significant $(p<0.05)$. Insulin was completely released from formulations of group B i.e., B1, B2 and B3 containing PVA and chitosan in different proportions and $\mathrm{C}$ group in which only PVA has been used during the first $15 \mathrm{~h}$ of the study. In contrast, only $35-50 \%$ of Insulin was released from A1, A2, A3 chitosan based microspheres for the same duration of time. The variance in release pattern may be attributed to the difference in the solubility of PVA and chitosan as the polymeric vehicles in the buffer solution $\mathrm{pH}$ 6.5. PVA is more water soluble than Chitosan in $\mathrm{pH} 6.5$ and its solubility depends on degree of polymerization and percentage of hydrolysis. For this reason the release rate of insulin from PVA microspheres is impressive. All the formulations showed burst release pattern from time 0 to $1 \mathrm{~h}$. Increasing the percentage of chitosan in formulations of group A, decreases the release rate of insulin from the microspheres which is not significant $(\mathrm{p}<0.05)$. The overall cumulative release of insulin from various group of formulations are shown in Figure 1. The physicochemical characteristics of the microspheres such as particle size, drug loading, 
percentage yield, MDT and recovery percentage were summarized also in Table 3. According to Table 3, decreasing the concentration of chitosan in group $\mathrm{B}$ formulation containing varying ratios of chitosan and PVA, decreases the particle size of the group B microspheres further $(\mathrm{p}<0.05)$. The size distribution of the microspheres differs. Application of $50 \%$ PVA in the polymeric blend leads to formation of microspheres with smaller mean particle size compared to PVA only containing polymeric microspheres. According to Figure 2a and Figure 2b, it is also evident that decreasing the concentration of chitosan in A and B groups of formulations, decreases the MDT magnitude, suggesting that the dissolution of group B which contains blend of chitosan and PVA is faster than group A containing chitosan alone. Among B group of formulations, B3 had the least MDT value which would be supported by the fact of prolonging drug release from the microspheres due to the desirable adhesion to the nasal mucosa. Presence of PVA in the structure of the microspheres, decreases the loading percentage of insulin from the vehicle $(p<0.05)$. To better understand the morphology of the microspheres, SEM images of the selected formulations was presented in Figure 3. According to Figure 3 , microspheres are spherical in shape, almost densely packed to each other. It is more evident in group $\mathrm{B}$ and $\mathrm{C}$ formulations, where PVA is present, more spherical microspheres have obtained. In chitosan only based microspheres, i.e., group A, decreasing the concentration of chitosan leads to the formation of more spherical shaped particles which are loosely packed. The formation of the spherical particles with the smooth surface increased by augmentation of chitosan concentration in deep particles structure. In group B formulations, increasing the ratio of chitosan in the polymeric blend increases the smoothness of the microspheres surface.

The blood plasma profiles of glucose concentration after intranasal administration of the A3 and B3 formulations are shown in Figure 4. It is well understood that, increasing the concentration of chitosan reduces the extent of insulin absorption via the intranasal rout in groups or rats. It would be justified by the fact that mucoadhesiveness and tight junction opening characteristics of chitosan molecules is responsible for the same phenomenon. These inherent properties of chitosan as a natural polymer have been reported previously. ${ }^{6}$ Polycationic structure and the positive surface charge of chitosan as a natural polymer could be the key element for this issue which has to be considered in large scale production of the formulation.

Table 3 Physical characteristic, release rate constant $(\mathrm{k})$, mean particles size, percentage of recovery in spray dryer, drug loading percentage, release efficacy percentage and mean dissolution time of groups $A, B$ and $C$ formulations

\begin{tabular}{|c|c|c|c|c|c|c|}
\hline Formulations & $\begin{array}{l}\text { Mean Particle Size }(\mu \mathrm{m}) \\
(\mathrm{mean} \pm \text { SD) }\end{array}$ & $\begin{array}{l}\text { Loading } \\
\%(\text { mean } \pm \text { SD) }\end{array}$ & Yield \% & $k($ mean $\pm S D)$ & $\begin{array}{l}\text { Release Efficacy } \\
\% \text { (mean } \pm \text { SD) }\end{array}$ & $\begin{array}{l}\text { Mean Dissolution Time } \\
\text { (h) (mean } \pm \text { SD) }\end{array}$ \\
\hline Al & $1.116 \pm 0.507$ & $4.3 \pm 02$ & $27.1 \pm 04$ & $0.148 \pm 0.009$ & $17.110 \pm 0.963$ & $41.168 \pm 1.860$ \\
\hline A2 & $2.083 \pm 0.664$ & $4.3 \pm 03$ & $27.1 \pm 02$ & $0.165 \pm 0.007$ & $20.803 \pm 0.963$ & $34.46 I \pm 1.104$ \\
\hline A3 & $1.666 \pm 0.752$ & $4.3 \pm 02$ & $27.18 \pm 02$ & $0.165 \pm 0.014$ & $21.290 \pm 0.963$ & $32.655 \pm 3.316$ \\
\hline $\mathrm{BI}$ & $1.166 \pm 0.408$ & $2.6 \pm 01$ & $25.31 \pm 03$ & $1.618 \pm 1.997$ & $43.693 \pm 0.963$ & $13.750 \pm 1.838$ \\
\hline B2 & $1.750 \pm 0.612$ & $2.6 \pm 02$ & $19.7 \pm 02$ & $0.306 \pm 0.203$ & $39.893 \pm 0.963$ & $14.740 \pm 1.335$ \\
\hline B3 & $1.50 \pm 0.547$ & $2.6 \pm 01$ & $12.65 \pm 0 \mid$ & $0.318 \pm 0.096$ & $42.639 \pm 0.963$ & $13.766 \pm 0.300$ \\
\hline C & $1.61 \pm 0.435$ & $2.2 \pm 02$ & $15.72 \pm 03$ & $0.578 \pm 0.506$ & $43.080 \pm 0.963$ & $|4.62| \pm 2.8 \mid$ \\
\hline
\end{tabular}

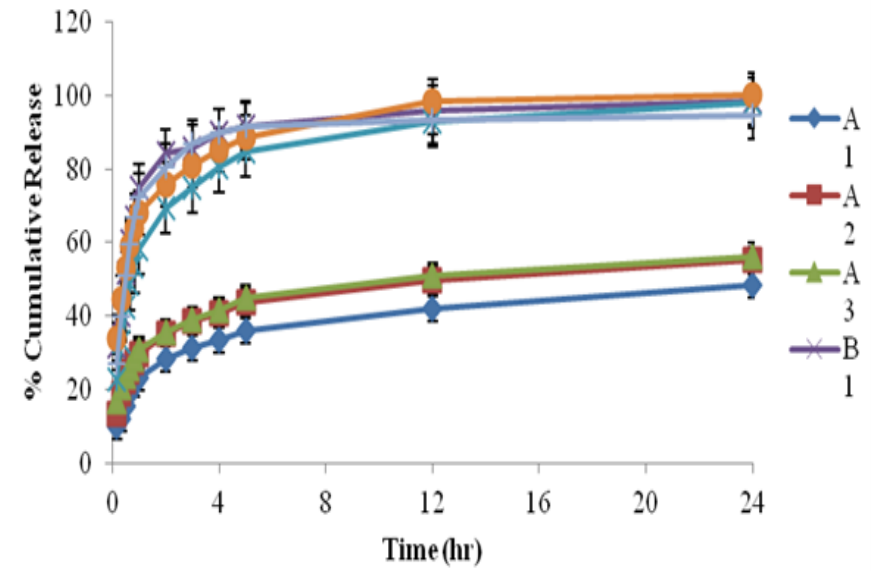

Figure I Overall cumulative percentage of insulin release from groups of A, $B$ and $C$ formulations.

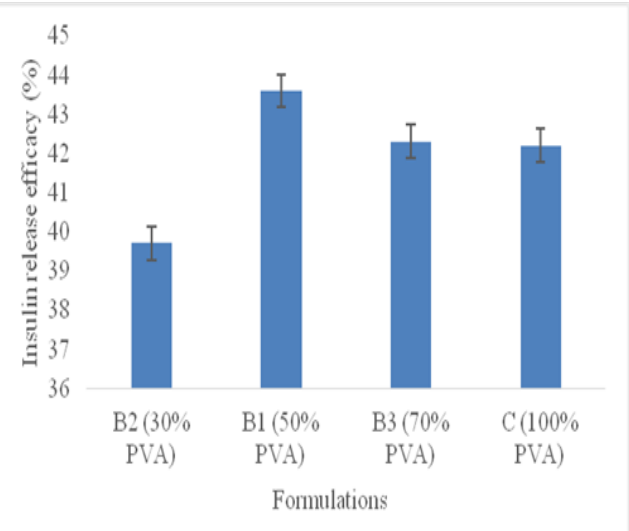

Figure 2a Insulin release efficacy of group B and C formulations with respect to PVA concentration and effect of PVA concentration on drug release from microspheres. 


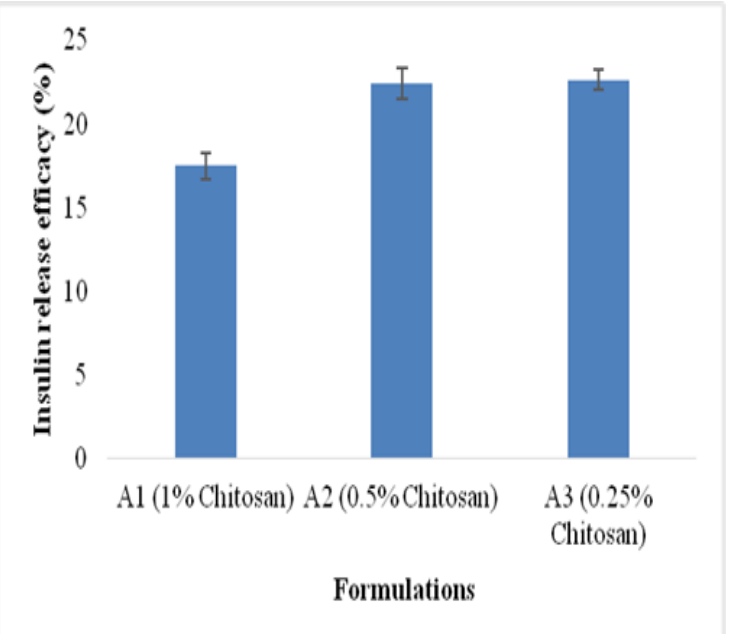

Figure $\mathbf{2 b}$ Insulin release efficacy of group $A$ formulations and the effect of chitosan concentration.

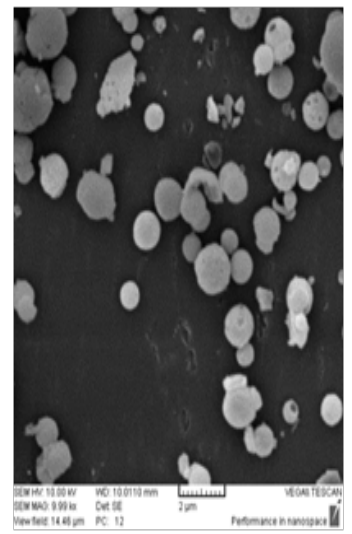

A2

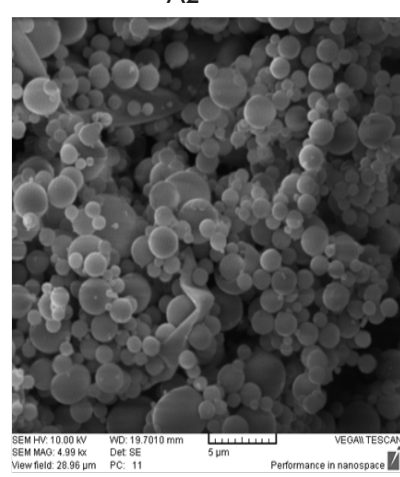

B2

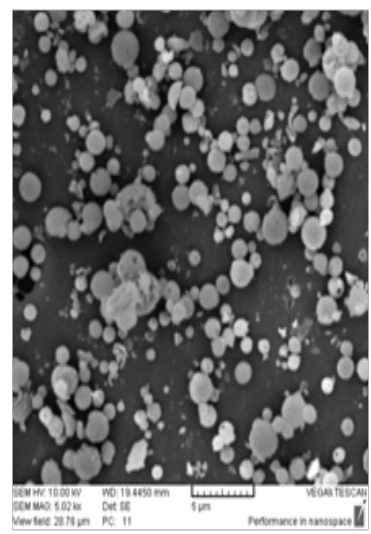

A3

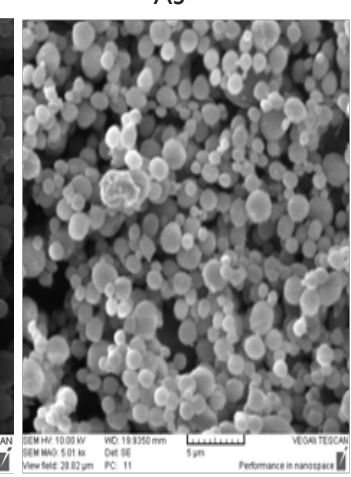

B3

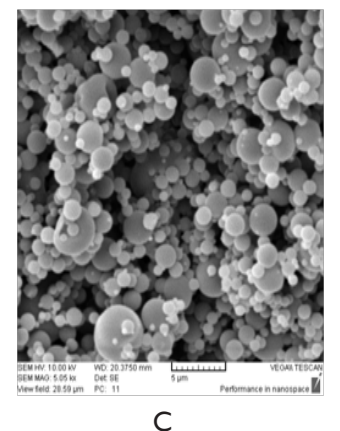

C

Figure 3 SEM images of selected formulations from group A, B and C.

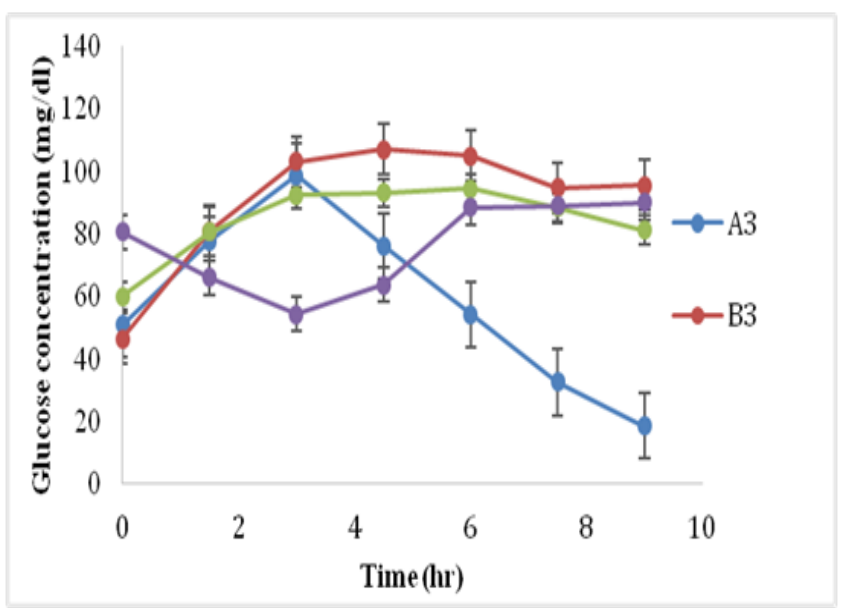

Figure 4 Glucose concentration $(\mathrm{mg} / \mathrm{dl})$ after intranasal administration of the formulations to control, insulin injection (standard) and optimized A3 and B3 formulations.

\section{Conclusion}

Based on this study, it was confirmed that the optimized composition of polymeric blend as well as manufacturing techniques used in the production of the microspheres in both small and large industrial levels are effective and determining factors on in vitro insulin release pattern and concerned physicochemical characteristics of the particles including rate and extent of drug release from the microspheres. These parameters have significant effect on in vivo behavior of the optimized formulations that directly affect the rate and the extent of absorption of insulin as a macromolecule drug moiety via intranasal rout of administration.

\section{Acknowledgements}

None.

\section{Conflict of interest}

The author declares no conflict of interest.

\section{References}

1. Arbit E. The Physiological Rationale for Oral Insulin Administration. Diabetes Technol Ther. 2004;6(4):510-517.

2. Fonte P, Araújo F, Reis S, et al. Oral Insulin Delivery:How Far are We? $J$ Diabetes Sci Technol. 2013;7(2):520-531.

3. Agrawal AK, Gupta PN, Khanna A, et al. Development and characterization of in situ gel system for nasal insulin delivery. Pharmazie. 2010;65(3):188-193.

4. Heinemann L, Pfützner A, Heise T. Alternative routes of administration as an approach to improve insulin therapy: Update on dermal, oral, nasal and pulmonary insulin delivery. Curr Pharm Des. 2001;7(14):13271351.

5. Arun Verma, Nitin Kumar, Rishabha Malviya, et al. Emerging Trends in Noninvasive Insulin Delivery. Journal of Pharmaceutics. 2014;9.

6. Sarkar MA. Drug metabolism in the nasal mucosa. Pharm Res. 1992;9(1):1-9.

7. Hayashi M, Hirasawa T, Muraoka T, et al. Comparison of water influx and sieving coefficient in rat jejunal, rectal and nasal absorptions of antipyrine. Chem Pharm Bull. 1985;33(5):2149-2152. 
8. Shorten PR, Mc Mahon CD, Soboleva TK. Insulin transport within skeletal muscle transverse tubule networks. Biophys J. 2007;93(9):30013007.

9. Costantino HR, Illum L, Brandt G, et al. Intranasal delivery:Physicochemical and therapeutic aspects. Int J Pharm. 2007;337(1-2):1-24.

10. Leary AC, Dowling M, Cussen K, et al. Pharmacokinetics and pharmacodynamics of intranasal insulin spray (Nasulin) administered to healthy male volunteers: Influence of the nasal cycle. J Diabetes Sci Technol. 2008;2(6):1054-1060.

11. Illum L. Nasal drug delivery-Recent developments and future prospects $J$ Control Release. 2012;161(2):254-263.

12. Maggio ET. Intravail:Highly effective intranasal delivery of peptide and protein drugs. Expert Opin Drug Deliv. 2006;3(4):529-539.

13. Cheng YH, Dyer AM, Jabbal Gill I, et al. Intranasal delivery of recombinant human growth hormone (somatropin) in sheep using chitosan-based powder formulations. Eur J Pharm Sci. 2005;26(1):9-15.

14. Illum L. Nanoparticulate systems for nasal delivery of drugs:A real improvement over simple systems? J Pharm Sci. 2007;96(3):473-483.

15. Al Ghananeem AM, Saeed H, Florence R, et al. Intranasal drug delivery of didanosine-loaded chitosan nanoparticles for brain targeting; an attractive route against infections caused by aids viruses. $J$ Drug Target. 2010;18(5):381-388.

16. Leary AC, Stote RM, Cussen K, et al. Pharmacokinetics and Pharmacodynamics of Intranasal Insulin Administered to Patients with Type 1 Diabetes: A Preliminary Study. Diabetes Technol Ther. 2006;8(1):81-88

17. Casettari L, Illum L. Chitosan in nasal delivery systems for therapeutic drugs. J Control Release. 2014;190:189-200.

18. Smith J, Wood E, Dornish M. Effect of Chitosan on Epithelial Cell Tight Junctions. Pharm Res. 2004;21(1):43-49.

19. Illum L, Watts P, Fisher AN, et al. Intranasal delivery of morphine. $J$ Pharmacol Exp Ther. 2002;301(1):391-400.
20. Alhalaweh A, Andersson S, Velaga SP. Preparation of zolmitriptan-chitosan microparticles by spray drying for nasal delivery. Eur J Pharm Sci. 2009;38(3):206-214

21. Abdel Mouez M, Zaki NM, Mansour S, et al. Bioavailability enhancement of verapamil $\mathrm{HCl}$ via intranasal chitosan microspheres. Eur $J$ Pharm Sci. 2014;51:59-66.

22. Gavini E, Rassu G, Muzzarelli C, et al. Spray-dried microspheres based on methylpyrrolidinone chitosan as new carrier for nasal administration of metoclopramide. Eur J Pharm Biopharm. 2008;68(2):245-252.

23. Dyer AM, Hinchcliffe M, Watts $P$, et al. Nasal delivery of insulin using novel chitosan based formulations:A comparative study in two animal models between simple chitosan formulations and chitosan nanoparticles. Pharm Res. 2002;19(7):998-1008.

24. Varshosaz J, Sadrai H, Alinagari R. Nasal delivery of insulin using chitosan microspheres. J Microencapsul. 2004;21(7):761-774.

25. Krauland AH, Leitner VM, Grabovac V, et al. In vivo evaluation of a nasal insulin delivery system based on thiolated chitosan. J Pharm Sci. 2006;95(11):2463-2472.

26. Illum L, Watts P, Fisher AN, et al. Novel chitosan-based delivery systems for the nasal administration of a LHRH-analogue. STP Pharma Sciences. 2002;10:89-94.

27. Arakawa Y, Kurokawa N, Maeda Y, et al. Change in solubility of insulin in therapeutic mixtures of insulin preparations:HPLC analysis. Diabetes Res Clin Pract. 1989;7(Suppl 1):93S-100S

28. Ortner K, Buchberger W, Himmelsbach M. Capillary electrokinetic chromatography of insulin and related synthetic analogues. J Chromatogr A. 2009;1216(14):2953-2957.

29. Oskar Wintersteiner, Harold A Abramson. The isoelectric point of insulin:electrical properties of adsorbed and crystalline insulin. J Biol Chem. 1933;99(3):741-753. 